\title{
Meningeal Melanocytoma
}

National Cancer Institute

\section{Source}

National Cancer Institute. Meningeal Melanocytoma. NCI Thesaurus. Code C4662.

A usually well differentiated melanocytic neoplasm arising from the meninges. It is characterized by the presence of epithelioid, fusiform, polyhedral, and spindle melanocytes without evidence of hemorrhage, necrosis, or high mitotic activity. Presenting symptoms include headache, vomiting, and neurological manifestations. Complete excision is usually curative. 